\title{
Response-Dependent Contributions of Human Primary Motor Cortex and Angular Gyrus to Manual and Perceptual Sequence Learning
}

\author{
Clive R. Rosenthal, ${ }^{1}$ Emma E. Roche-Kelly, ${ }^{2}$ Masud Husain, ${ }^{3}$ and Christopher Kennard ${ }^{1}$ \\ ${ }^{1}$ Department of Clinical Neurology, University of Oxford, Oxford, OX3 9DU, United Kingdom, ${ }^{2}$ Department of Clinical Neuroscience, Imperial College \\ London, London W6 8RP, United Kingdom, and ${ }^{3}$ Institute of Neurology, University College London, London WC1N 3BG, United Kingdom
}

\begin{abstract}
Motor sequence learning on the serial reaction time task involves the integration of response-, stimulus-, and effector-based information. Human primary motor cortex (M1) and the inferior parietal lobule (IPL) have been identified with supporting the learning of effectordependent and -independent information, respectively. Current neurocognitive data are, however, exclusively based on learning complex sequence information via perceptual-motor responses. Here, we investigated the effects of continuous theta-burst transcranial magnetic stimulation (cTBS)-induced disruption of M1 and the angular gyrus (AG) of the IPL on learning a probabilistic sequence via sequential perceptual-motor responses (experiment 1) or covert orienting of visuospatial attention (experiment 2). Functional effects on manual sequence learning were evident during 75\% of training trials in the cTBS M1 condition, whereas cTBS over the AG resulted in interference confined to a midpoint during the training phase. Posttraining direct (declarative) tests of sequence knowledge revealed that cTBS over M1 modulated the availability of newly acquired sequence knowledge, whereby sequence knowledge was implicit in the cTBS M1 condition but was available to conscious awareness in the cTBS AG and control conditions. In contrast, perceptual sequence learning was abolished in the perceptual cTBS AG condition, whereas learning was intact and available to conscious awareness in the cTBS M1 and control conditions. These results show that the right $\mathrm{AG}$ had a critical role in perceptual sequence learning, whereas M1 had a causal role in developing experience-dependent functional attributes relevant to conscious knowledge on manual but not perceptual sequence learning.
\end{abstract}

\section{Introduction}

Numerous cognitive faculties and behaviors, such as language acquisition, cognitive skill learning, and motor skill learning, can be conceptualized as a sequence learning process. Perceptualmotor aspects of sequence learning have been examined extensively using the serial reaction time task (SRT task), wherein sequential manual key presses confined to four fixed locations are performed in response to visual targets that appear at one of four corresponding spatial locations (Nissen and Bullemer, 1987; Shanks, 2005). Human functional neuroimaging studies indicate that a cortico-cerebellar network underlies sequence learning on the SRT task, with activation in the inferior parietal lobule (IPL) identified with coding an effector-independent (visual/spatial coordinates) description of successive locations (Grafton et al., 1998b; Seidler et al., 2005), whereas the coding of effector-specific (motor/response coordinates) information in egocentric space is identified with early activation within the primary motor cortex (M1) (Grafton et al., 1998b; Willingham, 1998). Current neuro-

\footnotetext{
Received May 20, 2009; revised July 17, 2009; accepted 0ct. 26, 2009.

This research was supported by a grant from the Wellcome Trust. We thank two anonymous reviewers for thoughtful comments on a previous version of this manuscript.

This article is freely available online through the J Neurosci Open Choice option.

Correspondence should be addressed to Dr. Clive Rosenthal, Department of Clinical Neurology, University of

0xford, Level 6, West Wing, John Radcliffe Hospital, 0xford, 0X3 9DU, UK. E-mail: clive.rosenthal@clneuro.ox.ac.uk. D01:10.1523/JNEUROSCI.2603-09.2009

Copyright $\odot 2009$ Society for Neuroscience $\quad$ 0270-6474/09/2915115-11\$15.00/0
}

cognitive data are, however, only informative about the neural areas related to learning complex sequence information involving perceptual-motor responses.

Recent behavioral evidence has shown that learning on the SRT task can proceed when responses are confined to sequential covert reorienting of visuospatial attention (Song et al., 2008). Hence, motor responses are not a necessary condition for complex sequence learning. This is an important consideration because task demands lead to different instantiations of the response-, stimulus-, and effector-based information that underlie sequence learning (Clegg et al., 1998). By extension, unless the contribution of the IPL to sequence learning can be shown to be independent of response mode, it is conceivable that activation within the IPL during manual sequence learning reflects the operation of more general mechanisms identified with the parietal cortex, such as perceptual-motor integration, task difficulty, or spatial attention (Willingham et al., 2002; Majerus et al., 2006). Here, we examine the role of the right angular gyrus (AG) - a subregion within the IPL-in sequence learning because it has been implicated in the maintenance and control of covert reorienting of attention over time within bilateral hemispaces, as part of a network supporting the detection of salient new items embedded in a sequence (Sack, 2009).

Given these limits on understanding the cortical substrate underlying sequence learning, we investigated the role of M1 and the right $\mathrm{AG}$ by administering continuous theta-burst transcra- 
nial magnetic stimulation (cTBS) (three pulses at $50 \mathrm{~Hz}$, repeated every $200 \mathrm{~ms}$ ) before training on the SRT task. We used the cTBS protocol because it induces disruption that outlasts the time of stimulation (up to $60 \mathrm{~min}$ ) and the effects are mediated by an analog of the type of long-term potentiation- and/or long-term depression-like changes in synaptic transmission that have been implicated in learning and memory (Ziemann et al., 2004; Huang et al., 2005, 2008; Cheeran et al., 2008). Responses on the SRT task involved either sequential unimanual key presses and free eye movements (experiment 1; manual condition) or covert reorienting of visuospatial attention (experiment 2; perceptual condition). Visual targets followed a probabilistic second-order conditional rule on which transition probabilities between spatial locations were unpredictable (improbable) on $15 \%$ of trials (Shanks et al., 2006). We exploited this stochastic property to measure the time course of the cTBS-induced effect on learning [reaction time (RT) to probable vs improbable trials] independently of general response execution and to encourage learning without awareness (implicit vs explicit learning). Learning and awareness were assessed using posttraining subjective, indirect (procedural) and direct (declarative) tests (Albouy et al., 2008). If the IPL is involved with coding effector-independent information related to learning per se, transient disruption should interfere with learning on the SRT task independently of the response mode. Conversely, transient disruption of the hypothesized effector-dependent contribution of M1 should affect learning on the manual, but not perceptual, SRT task.

\section{Materials and Methods \\ Participants}

Forty-eight participants (46 right-handed, 2 left-handed) (Oldfield, 1971) were recruited from the undergraduate student population at Imperial College London. Local research ethics committee approval was granted for the experimental procedure. All participants provided written informed consent and were screened to exclude those with a history of neurological disorder or head injury. Twenty-four participants took part in experiment 1 and 24 participants took part in experiment 2 . Within each experiment, the participants were subdivided into three groups according to whether cTBS was administered over the dominant hand area of the primary motor cortex ( $\left.\mathrm{CTBS} \mathrm{M} 1_{\mathrm{HAND}} ; n=8\right)$, the angular gyrus of the inferior parietal lobule (cTBS AG; $n=8$ ), or administered as sham cTBS over the vertex (electrode site, Cz; control; $n=8$ ). All participants had normal or corrected-to-normal visual acuity. None of the participants had previous experience of the SRT task or sequence learning tasks. All participants were naive to TMS.

\section{Design and materials}

Experiment 1 and experiment 2 each comprised three phases (Fig. 1): (1) magnetic resonance imaging (MRI)-guided neuronavigation and cTBS; (2) a training phase consisting of either a manual SRT task (experiment 1) or perceptual SRT task (experiment 2) performed alongside a vigilance task; and (3) a posttraining direct test phase comprising a sequence awareness questionnaire (Curran, 1997), a manual (experiment 1) or perceptual (experiment 2) cued-generation task, and a manual (experiment 1) or perceptual (experiment 2) recognition test. Conjoint performance across the subjective, indirect (procedural) and direct (declarative) tests administered during phase 3 was used to detect learning and, importantly, to be highly sensitive to the extent to which knowledge was primarily implicit or explicit (Destrebecqz and Cleeremans, 2001). All of the behavioral tasks were implemented using a proprietary program written in $\mathrm{C}++$.

Phase 2. Training on experiments 1 and 2 involved a 3 (site of stimulation: cTBS $\mathrm{M}_{\mathrm{HAND}}$, cTBS AG, control) $\times 12$ (training blocks: 12) mixed-factorial design, with site as a between-subjects factor and block as a within-subjects factor. Each block of the manual SRT task and percep- tual SRT task comprised 100 trials. Twelve training blocks were administered after an initial pseudorandom practice sequence. In each block, ninety-six trials were critical items and the first four trials were buffers. Two basic sequences were generated in accordance with a second-order conditional (SOC) deterministic rule, such that each target location was determined on the basis of the two preceding targets (Reed and Johnson, 1994) (Fig. 1, phase 2). These sequences-SOC1 and SOC2-were modified so that the assigned training sequence appeared according to the target second-order transitions with a probability 0.85 and were replaced by second-order transitions from an alternate sequence with a probability 0.15 . Hence, there were short- and longer-range predictive target locations - a probable/improbable ratio of 0.85:0.15 can be learned to asymptote after 9-12 blocks of trials (Shanks et al., 2006). Each block of the 96 critical trials began at a random point in the primary sequence. Target sequences were counterbalanced across participants.

Visual stimuli comprised two sizes of target-a standard target $(5 \mathrm{~mm}$ diameter) and a large-diameter target (LDT) $(8 \mathrm{~mm}$ diameter $)$ - that appeared in one of four squares $(35 \times 35 \mathrm{~mm})$ arranged along the horizontal meridian against a white background. Visual stimuli were presented on a 21 inch Sony Trinitron cathode ray tube computer monitor configured to a screen resolution of $1024 \times 769$ pixels at a $100 \mathrm{~Hz}$ refresh rate, positioned at a $75 \mathrm{~cm}$ viewing distance. Screen locations of the squares corresponded with positions $1-4$ as read from left to right, with the spatial locations corresponding to numeric values of the probabilistic SOC sequence. In each block of the manual and perceptual SRT task, LDTs were presented between 18 and $36 \%$ of trials; the order of the LDTs was random within a block and set at a proportion to ensure that participants performed at ceiling.

Phase 3. Direct tests of sequence knowledge immediately followed the SRT task and were administered in the same response mode as the training phase (Fig. 1). The sequence awareness questionnaire comprised five propositions (Curran, 1997). A novel cued-generation task followed the sequence awareness questionnaire and comprised an inclusion test (I) and an exclusion test (E). Test order was counterbalanced across participants. Stimulus materials on each trial were generated by starting at each ordinal position of SOC1 and SOC2 for two consecutive positions. Sequence knowledge on the cued-generation task is inferred if follow-on self-generated responses on the inclusion test and/or exclusion test exceed baseline (B) (for a full specification of the baseline, see Results). Intentional, conscious control of acquired sequence knowledge is inferred if participants are able to respond in accordance with test instructions (i.e., I trained $>\mathrm{E}$ trained; $\mathrm{E}$ trained $=\mathrm{B}$ ). In contrast, a null effect (i.e., I trained $=\mathrm{E}$ trained, or $\mathrm{E}$ trained $>\mathrm{B}$ ) is construed as evidence of implicit knowledge (Destrebecqz and Cleeremans, 2001).

The recognition test followed the cued-generation task and comprised 12 trained (old) and 12 untrained (new) six-element sequences. Twelve sequences (starting from each ordinal position of the 12-element SOC sequence for six consecutive locations) were generated from SOC1 and 12 were generated from SOC2. To ensure sensitivity to even partial declarative knowledge, a six-point scale was used to obtain a confidence rating for each six-element sequence (Shanks and Johnstone, 1999). Manual recognition test performance was also examined for response priming to trained (vs untrained) sequences because recognition is dependent on explicit knowledge and on the processing fluency associated with performing each six-element sequence (Shanks and Perruchet, 2002).

\section{MRI-guided neuronavigation and cTBS}

All participants in the M1 and AG cTBS groups underwent an MRI anatomical scan. Scans were obtained in anterior commissural-posterior commissural orientation, using high-resolution (256 slices, $1 \mathrm{~mm}$ apart), axial T1-weighted (magnetization-prepared rapid-acquisition gradient echo) images. Scans were acquired using a $1.5 \mathrm{~T}$ clinical scanner fitted with an echo-planar imaging upgrade (Siemens).

TMS coil positioning was guided using optical tracking via the frameless stereotactic system (BrainSight; Rogue Research). MRI neuronavigation was used to reduce the potential impact that low statistical power can have on being able to reject the null (Sack et al., 2009). In the cTBS AG condition, the right AG was identified within the posterior region of 


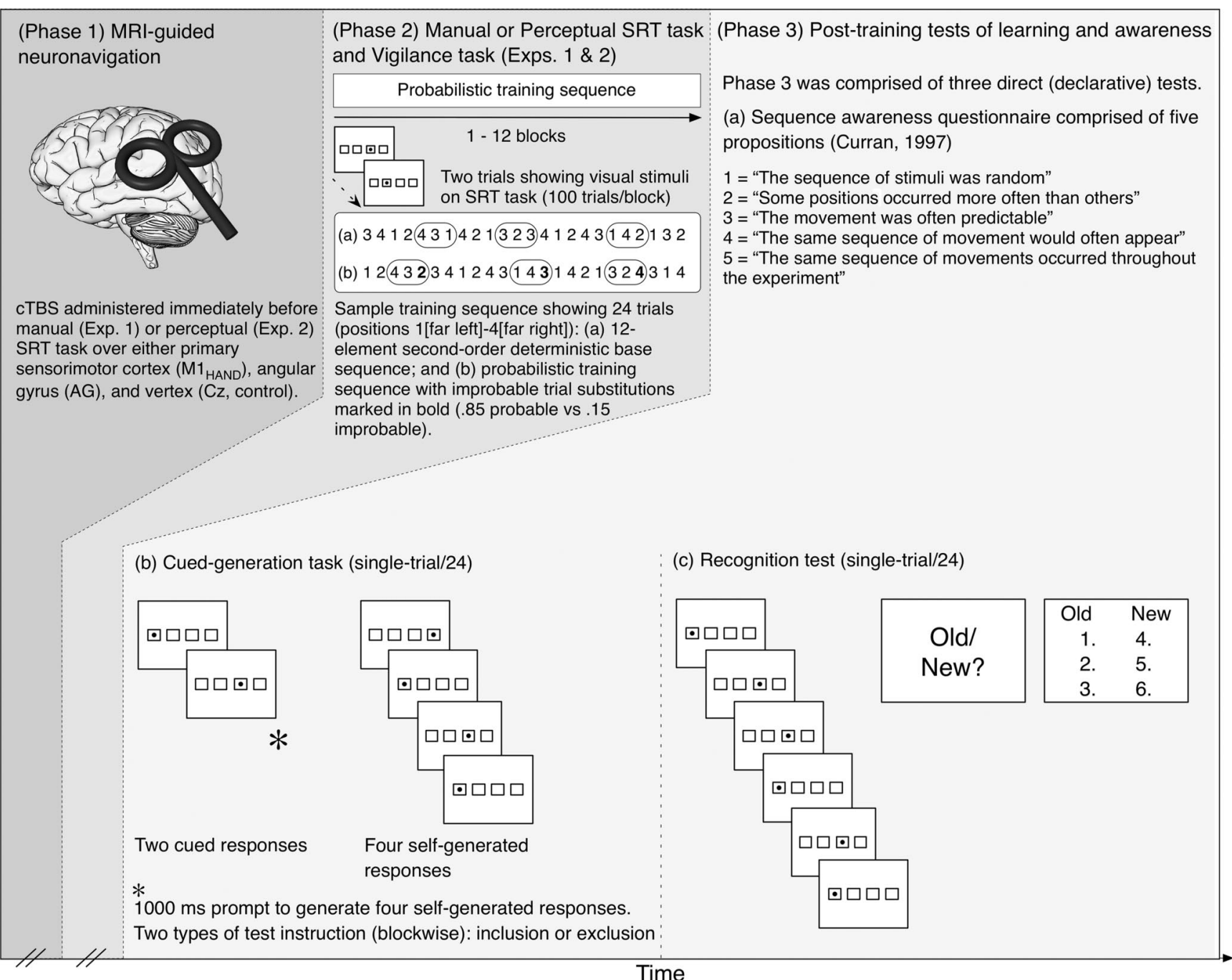

Time

Figure 1. Experimental setup and design in manual (experiment 1) and perceptual (experiment 2) sequence learning. Phase 1, MRI-guided neuronavigation and continuous theta-burst transcranial magnetic stimulation. TMS coil positioning was guided using optical tracking via a frameless stereotactic system in conjunction with an anatomical MRI scan. Phase 2, Training phase: each block of trials followed a probabilistic second-order conditional sequence. Participants were instructed to respond with a location contingent manual key press to each visual target (experiment 1: manual SRT task) or maintain fixation and attend to each target (experiment 2: perceptual SRT task). To ensure that participants attended to the sequence (experiments 1 and 2), they were also instructed to maintain a blockwise cumulative count of the number of large-diameter target presented among the standard targets and report the value at the end of each block. Phase 3, Posttraining direct tests of sequence knowledge: $\boldsymbol{a}$, sequence awareness questionnaire; $\boldsymbol{b}$, schematic of the cued-generation task showing one trial comprising two cues and four self-generated responses. Participants were instructed to respond to the two cues in the same way as on the SRT task. Next, either a red (exclusion test) or green (inclusion test) question mark appeared in each square (blockwise) as the prompt to self-generate four manual (experiment 1) or four vocal (experiment 2) responses that were either different from (exclusion test) or the same as (inclusion test) the training sequence; and c, schematic of the recognition test showing one trial comprising a six-element sequence derived from the trained (old) sequence or an untrained (new) sequence presented in same way as on the respective SRT tasks. Participants determined whether sequence was old/seen or new/unseen and then rated their confidence on a six-point scale.

the IPL as the area directly adjacent to the dorsolateral projection of the superior temporal sulcus; the superior temporal sulcus bifurcates the AG. The AG was localized for each participant in slice and three-dimensionalrendered T1-weighted MRI brain scans using a frameless stereotaxy system (BrainSight; Rogue Research). These data were used to coregister the neuroanatomical site with the scalp surface. Stimulation was administered using a figure-of-eight coil (70 $\mathrm{mm}$ diameter) connected to a Magstim Super Rapid stimulator.

The $M 1_{\text {HAND }}$ site of stimulation was selected as the site that evoked maximum movement in the hand contralateral to the site of stimulation at the lowest level of stimulator output. Individual resting motor threshold (RMT) for $\mathrm{M}_{\text {HAND }}$, as a measure of M1 stimulation (Robertson et al., 2003), was used to determine stimulator output for cTBS in the AG and $\mathrm{M}_{\mathrm{HAND}}$ conditions. RMT was established as the lowest output intensity of single-pulse TMS to elicit at least three consecutive movements in the contralateral relaxed hand after five pulses of TMS. cTBS [trains of three pulses at $50 \mathrm{~Hz}$ repeated at $200 \mathrm{~ms}$ intervals (i.e., at $5 \mathrm{~Hz}$ ) (Huang et al., 2005)] was applied at 70\% of RMT immediately before training on the SRT task. In experiment 1 , there was no significant difference in the mean stimulator output between cTBS $\mathrm{M1}_{\mathrm{HAND}}$ (mean of 44) and AG (mean of 44$)$ conditions $\left(t_{(14)}=0.14, p=0.89\right.$ ); correspondingly, in experiment 2 , there was no significant difference in the mean stimulator output between the cTBS M1 $1_{\text {HAND }}$ (mean of 44) and AG (mean of 48) conditions $\left(t_{(14)}=0.99, p=0.33\right)$.

In experiment 1, cTBS was applied for a single continuous $20 \mathrm{~s}$ train, whereas in experiment 2, cTBS was applied for $30 \mathrm{~s}$; the longer duration was intended to address an anticipated increase in the training time when recording eye movements on the perceptual SRT task. In the control condition, a commercially available double $70 \mathrm{~mm}$ placebo coil system positioned over the $\mathrm{Cz}$ electrode vertex position was used to administer stimulation at the same frequency as used with the cTBS conditions (Magstim). The coil replicates the appearance and operation of the standard double $70 \mathrm{~mm}$ coil used in the AG and M1 conditions and provides mild cutaneous stimulation and discharge noise without stimulating cor- 
tical tissue. An IBM personal computer-compatible computer running E-Prime software (version 1.1; Psychology Software Tools) controlled the delivery of cTBS. Participants wore a close-fitting nylon scalp cap to enable marking of the regions on the scalp designated to overlie the $\mathrm{M}_{\text {HAND }}$ area, $\mathrm{AG}$, and the vertex control site ( $\mathrm{Cz}$ electrode position) (Jasper, 1958).

\section{Eye movement recording}

Eye movements were recorded using an Eyelink II infrared eye tracker to ensure continuous central fixation during each block of the perceptual SRT task and perceptual direct tests (spatial resolution, $<0.5^{\circ}$; SR Research). Eye movements were monitored in real time by the experimenter to ensure that participants maintained central fixation. Head movements were minimized using a chin rest. Verbal feedback was provided if a participant deviated from central fixation. Viewing was binocular. Monocular, pupil-only eye tracking of the dominant eye was conducted at a sampling rate of $250 \mathrm{~Hz}$. Calibration and validation of eye position was performed using a five-element array. Eye movement fixation data were analyzed offline using a proprietary application developed within MATLAB version 6.5.1 (MathWorks). Saccades with latency of 12 $\mathrm{ms}$ or less were excluded from a trial sample, as were saccades that began and ended within a central region $\left(1^{\circ}\right)$. Less than $7 \%$ of trials in the perceptual cTBS $\mathrm{M}_{\mathrm{HAND}}$, $\mathrm{CTBS} \mathrm{AG}$, and control conditions were associated with saccades, which indicates that participants were able to maintain central fixation.

\section{Behavioral tasks}

Participants were tested individually, and both experiments were performed in a dark visual Ganzfeld. An experimental session lasted $\sim 1 \mathrm{~h} 30$ min in experiment 1 and $1 \mathrm{~h} 45 \mathrm{~min}$ in experiment 2 .

Manual and perceptual SRT tasks (phase 2). Each trial of the SRT task comprised a single target stimulus presented at one of four possible locations. On the manual SRT task, participants were instructed to press one of four keys in response to target onset (RB Series Response Pad, model RB-730; Cedrus Corporation) and were allowed to make free eye movements. Keys were marked 1, 2, 3, and 4 and corresponded to screen locations 1 (left) to 4 (right). Responses in the manual SRT task were made to locations 1-4 with the digitus secundus manus, digitus medius, digitus annularis, and digitus minimus manus of the dominant hand, respectively. On detection of a manual response, the target stimulus was extinguished and the next trial started, or, if no response was detected, the target was extinguished after $3000 \mathrm{~ms}$. On the perceptual SRT task, participants were instructed to maintain central fixation on a circle $(0.3$ $\mathrm{cm}$ diameter) positioned midway between locations 2 and 3. Each trial began with the presentation of a target stimulus at the center of one of the four squares (1000 ms). Extinction of the target was followed by a $200 \mathrm{~ms}$ interval. Hence, peripheral targets captured attention in an exogenous and automatic manner, as a component of the endogenous allocation of attention (Juola et al., 1995).

Vigilance task. Alongside the manual and perceptual SRT tasks, participants were instructed to maintain a cumulative count of the number of LDTs presented during each block and report the value at the end of each block. The vigilance task was important for ensuring that participants attended to the sequence of visual stimuli, particularly on the perceptual SRT task. On-screen feedback on vigilance task performance was based on accuracy relative to the actual number of LDTs: participants entering a count within $5 \%$ accuracy were informed that their count was accurate and were asked to continue with their good performance; participants responding with count of $5 \%$ error or greater were shown their percentage underestimation or overestimation and were instructed to try harder in the forthcoming block of trials.

Posttraining direct tests of learning and awareness (phase 3). After selecting a response on the sequence awareness questionnaire to indicate the extent to which regularities had been detected during the training session, participants were informed that the target stimuli had followed a regular repeating sequence during the training session. Each trial of the cued-generation task (manual and perceptual) involved the presentation of two consecutive cue stimuli on the computer screen (Fig. 1, phase 3b). Participants were instructed to either respond with a manual key press (experiment 1; manual cued-generation task) or attend to each cue (experiment 2; perceptual cued-generation task) and then use knowledge acquired on the SRT task to either enter four key presses (experiment 1) or vocalize (experiment 2 ) four locations that followed on from the twocue sequence in accordance the test instructions. Instructions on the inclusion test directed participants to respond with the next four positions of the training sequence, even if they felt they could not remember particular parts of the sequence that followed the cues. On the exclusion test, participants were asked to respond with a sequence that was novel and avoided the training sequence. Participants were reminded to avoid responding with contiguous repetitions of a single location or natural sequences because these were not presented during training (e.g., 4-4-2-1 or 1-2-3-4). On the perceptual cued-generation task, the experimenter entered a key press contingent with each vocal response. All selfgenerated responses led to a black circle appearing in a corresponding target location. After completion of each cued-generation test, participants were asked to indicate their confidence, on a scale of $0-100$, with responding in accordance with the test instructions. Responses to each six-element sequence on the recognition test were performed in the same way as on the SRT task (Fig. 1, phase 3c). After responding to each sixelement sequence, participants were asked to determine whether the sequence was "old/seen" or "new/unseen"; that is, participants were asked to decide whether or not the sequence had appeared during the training session. After each old/new discrimination, participants were asked to rate how confident they were in their judgment on the six-point scale.

\section{Results}

\section{Experiment 1}

Participants in all three between-subject conditions achieved $>95 \%$ accuracy on the vigilance task, which indicates that participants were able to attend consistently to the target sequence and could reliably discriminate LDTs from standard targets. Mean end times in the cTBS AG, cTBS M1 $1_{\text {HAND }}$, and control conditions were $32 \mathrm{~min} 57 \mathrm{~s}$, $31 \mathrm{~min} 52 \mathrm{~s}$, and $33 \mathrm{~min} 12 \mathrm{~s}$, respectively.

\section{Response execution and learning on the manual SRT task}

Mean error rates collapsed across block were $<1 \%$ for each of the between-subject conditions. A 3 (site: $\mathrm{M} 1_{\mathrm{HAND}}, \mathrm{AG}$, control) $\times$ 12 (block: 12) mixed-factorial ANOVA performed on the mean SRT task error rates revealed no significant main effects or interactions $(F$ values $<1.2)$. Hence, there were no systematic differences in error as a function of site of stimulation or block. This level of error on the SRT task is well within level of response accuracy routinely reported under single-task conditions. Manual RTs for the first four buffer trials of each block and error trials were excluded from the analyses performed on the latency data. To assess the effect of training, RTs were first summarized by computing the mean RTs in each block to probable and improbable trials for each participant. Figure $2 a$ shows the mean blockwise learning scores (mean RT to probable trials - mean RT improbable trials) obtained over the manual SRT task, plotted separately for the cTBS $\mathrm{M1}_{\mathrm{HAND}}$, $\mathrm{cTBS} \mathrm{AG}$, and control conditions. A negative score that is significantly different from 0 is consistent with priming. A 3 (site: $\mathrm{M}_{\mathrm{HAND}}, \mathrm{AG}$, control) $\times 2$ (sequence: probable, improbable) $\times 12$ (block: 12) mixedfactorial ANOVA performed on the mean RTs revealed a significant main effect of sequence $\left(F_{(1,21)}=23.32, p<0.0001\right)$ and block $\left(F_{(11,231)}=4.89, p<0.00001\right)$. Importantly, although there was no significant main effect of site $\left(F_{(2,21)}=2.13, p=0.14\right)$, there was a significant interaction between site and sequence $\left(F_{(2,21)}=5.37, p<0.01\right)$, which indicates that emergence of sequence knowledge differed between groups. All other interaction terms were not significant $(F$ values $<1.4$ ).

Figure $2 b$ shows the emergence of sequence knowledge as a function of the mean end times for each block of the manual 


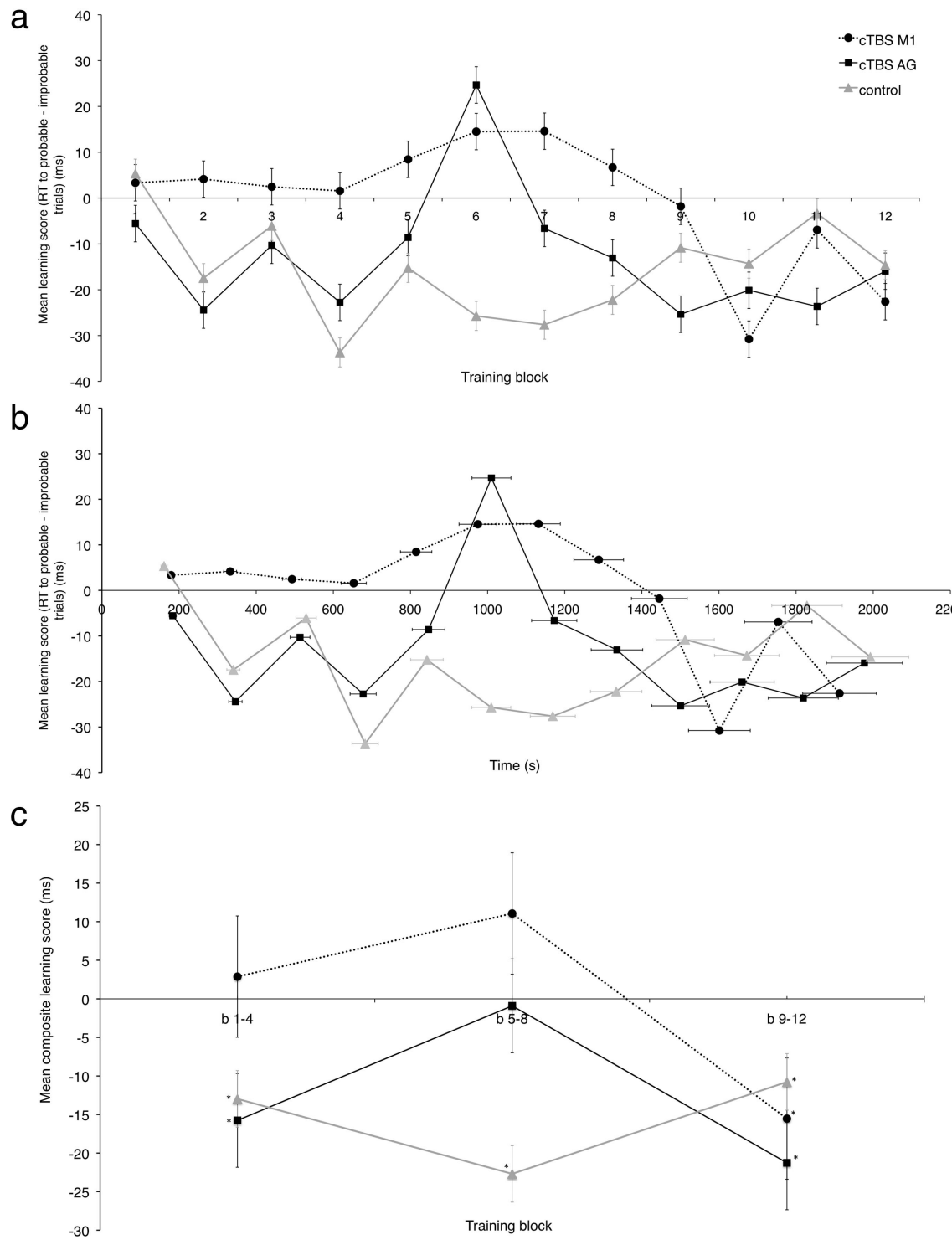

Figure 2. Manual sequence learning (experiment 1). $\boldsymbol{a}$, Mean blockwise learning scores (RT to probable trials - RT to improbable trials) across 12 blocks of the manual SRT task, plotted separately for each of three site conditions ( $M 1_{H A N D}, A G$, and control), with SE. Probabilistic sequence SOC1 (S1) was used for half of the participants, and probabilistic sequence SOC2 (S2) was used for the remaining half of the participants on blocks $1-12$. The nontrained SOC was used on improbable trials. $\boldsymbol{b}$, Mean learning scores (RT to probable trials - RT to improbable trials) on the manual SRT task plotted against the mean end times (seconds) for each block and shown separately for each of three site conditions ( $\mathrm{M1}_{\mathrm{HAND}}, \mathrm{AG}$, and control), with SE; ${ }^{*} p<0.05$. c, Means of composite learning scores (probable - improbable trials) calculated to summarize the learning effects for three training epochs (blocks 1-4, 5-8, and 9-12) and plotted separately each site of stimulation, with SE.

SRT task. A 3 (site: $M 1_{\text {HAND }}, A G$, control) $\times 12$ (block: 12 ) mixed-factorial ANOVA performed on the mean block end times revealed a significant main effect of block $\left(F_{(11,231)}=6121.7, p<\right.$ $0.00001)$ and no main effect of site $\left(F_{(2,21)}=1.01, p=0.38\right)$, and, importantly, there was no interaction between site and block $\left(F_{(22,231)}=1.36, p=0.13\right)$. Hence, the differences in the emergence of sequence knowledge between each site cannot be readily attributed to systematic differences in the time course underlying training in each between-subjects condition.

To summarize the effects of cTBS on learning at each site as a function of exposure to the sequence, a measure of learning across blocks was calculated by obtaining a composite learning score (mean RT to probable trials - mean RT improbable trials) for epochs at the beginning (blocks 1-4), middle (blocks 5-8), and end (blocks 9-12) of the manual SRT task (Fig. 2b). Mean learning scores for the three epochs are shown in Figure $2 c$. A 3 (site: $\mathrm{M}_{\mathrm{HAND}}$, AG, control) $\times 3$ (epoch: early, middle, late) mixed-factorial ANOVA performed on the mean composite learning scores revealed significant main effects of site and epoch $\left(F_{(2,21)}=5.37, p<0.05 ; F_{(2,42)}=\right.$ $3.33, p<0.05$, respectively) and a significant interaction between site and epoch $\left(F_{(4,42)}=3.71, p<0.05\right)$. The latter interaction indicates a difference in the magnitude of the learning effect between the three groups as a function of exposure to the sequence. Hence, despite the similar blockwise time points, learning proceeded at a different rate in the three groups.

Repeated measures $t$ tests performed against chance (0) revealed significant learning at early, middle, and late epochs in the control condition $\left(t_{(7)}=2.85,3.41\right.$, and 2.28, $p$ values $<0.05)$, whereas in the cTBS $M 1_{\text {HAND }}$ condition, learning was only significant in the late epoch $\left(t_{(7)}=\right.$ $3.57, p<0.01$ ). RTs associated with probable and improbable transition locations in the cTBS $\mathrm{M} 1_{\text {HAND }}$ condition were equivalent until block 10: block 9 mean end time, 23 min $52 \mathrm{~s}$; block 10 mean start and end times, $24 \mathrm{~min} 18 \mathrm{~s}$ and $26 \mathrm{~min}$ $40 \mathrm{~s}$, respectively. In cTBS AG condition, there was significant learning in the early $\left(t_{(7)}=2.41, p<0.05\right)$ and late $\left(t_{(7)}=3.39\right.$, $p<0.01)$ epochs, whereas the learning score was not significantly different from 0 in the middle epoch (mean start and end times, $11 \mathrm{~min} 31 \mathrm{~s}$ and $22 \mathrm{~min} 15 \mathrm{~s}$, respectively) $\left(t_{(7)}=0.24, p=0.82\right)$. The loss of sensitivity to improbable trials was particularly pronounced at block 6: block 6 mean start and end times, $14 \mathrm{~min} 17 \mathrm{~s}$ and $16 \mathrm{~min} 50 \mathrm{~s}$, respectively. Overall, evidence of a functional-temporal dissociation between the cTBS $M 1_{\text {HAND }}$ and cTBS AG conditions combined with the results from control condition suggests that the functional effects were not attributable to nonspecific effects associated with TMS or attributable to systematic between-group differences in the time points related to each block end time.

Effects of cTBS over M1 and AG on posttraining direct tests of learning and awareness

Subjective measures of sequence learning. Participants in the cTBS $\mathrm{M}_{\mathrm{HAND}}$ (mean $\pm \mathrm{SE}, 1.9 \pm 0.45$ ) and control (mean $\pm \mathrm{SE}, 1.5 \pm$ 0.45 ) conditions perceived the movement of target stimuli to be essentially random, whereas participants in the cTBS AG condition (mean $\pm \mathrm{SE}, 2.8 \pm 0.45$ ) were aware that some positions occurred more often than others, but there was no significant difference between the three groups (Kruskal-Wallis ANOVA, $\left.\chi_{(2)}^{2}=2.41, p=0.30\right)$. We also assessed participants' subjective rating of their performance on the cued-generation task. Participants in all between-subjects conditions were more confident in their ability to generate a novel sequence on the exclusion test 
(mean \pm SE, $51.4 \pm 4.75$ ) than generate the trained sequence on the inclusion test $($ mean \pm SE, $24.6 \pm 3.85)\left(F_{(1,21)}=19.10\right.$, $p<0.001)$. No interactions were significant $(F$ values $<1.8)$.

Cued-generation task. Mean start times for the cued-generation task in the cTBS $\mathrm{M} 1_{\mathrm{HAND}}$, cTBS AG, and control conditions were $37 \mathrm{~min} 4 \mathrm{~s}, 37 \mathrm{~min} 3 \mathrm{~s}$, and 37 min $46 \mathrm{~s}$, respectively. Performance was assessed by first calculating the proportion of SOC sequence triplets generated out of the maximum number of correct SOC triplets that could be generated. Four possible triplets could be generated on each of the 12 trials per test, thereby yielding a possible 48 correct SOC triplets on each test. Baseline rates of responding were calculated separately for each test as the proportion of triplets that were inconsistent with the training sequence, i.e., the value was the proportion of triplets that corresponded to the untrained sequence on each free-generation test (Wilkinson and Jahanshahi, 2007).

Figure $3 a$ shows the mean proportions of trained and baseline SOCs generated under each site condition for the inclusion and exclusion tests. A 3 (site: $\mathrm{M1}_{\mathrm{HAND}}, \mathrm{AG}$, control) $\times 2$ (test: inclusion, exclusion $) \times 2$ (sequence: trained, baseline) mixed-factorial ANOVA revealed significant main effects of test $\left(F_{(1,21)}=9.99\right.$, $p<0.01)$ and sequence $\left(F_{(1,21)}=20.74, p<0.001\right)$. The interaction between site, test, and sequence was also significant $\left(F_{(2,21)}=\right.$ $5.17, p<0.05)$. No other effects were significant. Planned comparisons revealed that participants in the cTBS AG and control conditions were able to exert intentional, conscious control when responding on the cued-generation task (i.e., I trained $>\mathrm{E}$ trained) $\left(F_{(1,21)}=14.02, p<0.01\right.$ and $F_{(1,21)}=8.00, p<0.01$, respectively), whereas the generation of trained sequences was equivalent across inclusion and exclusion tests in the cTBS M1 HAND condition $(F<1)$. Importantly, participants in the cTBS AG condition generated the trained sequence above baseline in the inclusion test $\left(F_{(1,21)}=8.77, p<0.01\right)$ and were able to exclude the trained sequence from responses on the exclusion test $\left(F_{(1,21)}\right.$ $=0.35, p=0.56$ ) (i.e., $\mathrm{E}$ trained $=\mathrm{B}$ ). Conversely, participants in the cTBS M1 $1_{\mathrm{HAND}}$ condition were unable to avoid generating the trained sequence above baseline on the exclusion test $\left(F_{(1,21)}=\right.$ $15.02, p<0.001)$, whereas generation of the trained sequences on the inclusion test was equivalent to baseline $\left(F_{(1,21)}=1.28, p=\right.$ $0.28)$. Participants in the control condition generated the trained sequence above baseline on the inclusion test $\left(F_{(1,21)}=8.77, p<\right.$ $0.01)$ and were able to refrain from generating the trained sequence on the exclusion test $(F<1)$. Collectively, these results reveal that sequence knowledge in the manual $\mathrm{cTBS} M 1_{\mathrm{HAND}}$ condition was implicit ( $\mathrm{I}=\mathrm{E}$ trained; $\mathrm{E}$ trained $>\mathrm{B}$ ), whereas sequence knowledge was available to conscious, intentional control in the CTBS AG and control conditions (I trained $>$ E trained; I trained $>\mathrm{B}$; E trained $=\mathrm{B}$ ). All planned comparisons were significant for Bonferroni-Holm corrected $p$ values.

Recognition test confidence ratings and recognition priming. Mean start times on the recognition test in the cTBS $M 1_{\text {HAND }}$, cTBS AG, and control conditions were $45 \min 7 \mathrm{~s}, 45 \min 34 \mathrm{~s}$, and 46 min $2 \mathrm{~s}$, respectively. Results from the mean recognition confidence ratings to trained and untrained sequences were consistent with the conclusions drawn from the cued-generation task
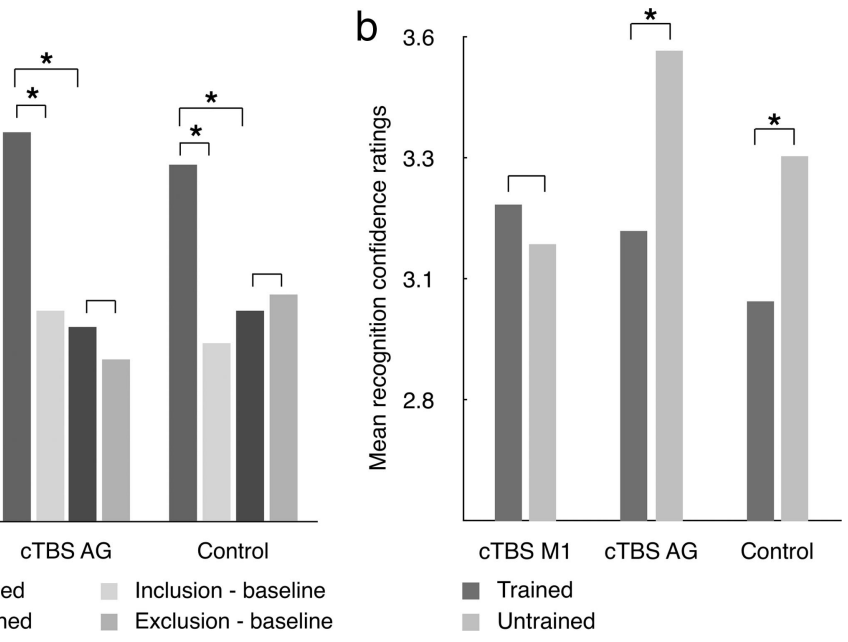

Figure 3. Manual sequence learning (experiment 1). $\boldsymbol{a}$, Mean proportions of trained and baseline $S O C$ triplets generated for assigned to the 24 six-item short sequences for each condition. Trained sequences were allocated a rating between 1 and

(Fig. $3 b$, lower ratings correspond to greater confidence that the sequence was "old"). A 3 (site: $\mathrm{M}_{\mathrm{HAND}}, \mathrm{AG}$, control) $\times 2$ (sequence: trained, untrained) mixed-factorial ANOVA revealed a significant main effect of sequence $\left(F_{(1,21)}=7.26, p<0.05\right)$ and a significant interaction between site and sequence $\left(F_{(1,21)}=3.78\right.$, $p<0.05)$. No other effects were significant. Planned comparisons based on mean confidence ratings revealed that participants were able to discriminate between trained and untrained sequences in the cTBS AG and control conditions $\left(F_{(1,21)}=8.49, p<0.01\right.$ and $F_{(1,21)}=5.87, p<0.05$, respectively), whereas in the cTBS $M 1_{\text {HAND }}$ condition, participants were unable to recognize the trained sequence $(F<1)$.

Examination of RTs to trained and untrained sequences can provide insight into whether there is evidence of priming of the trained sequence in the absence of recognition (Shanks et al., 2006) (Fig. 4). A 3 (site: $\mathrm{M}_{\mathrm{HAND}}, \mathrm{AG}$, control) $\times 2$ (sequence: trained, untrained) $\times 6$ (position: 1-6) mixed-factorial ANOVA performed on the mean recognition RTs at each position revealed significant main effects of sequence $\left(F_{(1,21)}=8.41, p<0.01\right)$ and position $\left(F_{(5,105)}=186.84, p<0.0001\right)$. Interactions between site and sequence $\left(F_{(2,21)}=8.47, p<0.01\right)$, between site and position $\left(F_{(10,105)}=2.74, p<0.01\right)$, and between site, sequence, and position $\left(F_{(10,105)}=2.70, p<0.01\right)$ were all significant. No other main effects or interactions were significant. Because positions 3-6 were predictable from the preceding targets (i.e., for trained sequences) and positions 1-2 were not predictable, position 3 is the first trial on which priming of the trained sequence can be detected as faster latency of response (Shanks et al., 2006). Figure $4 b$ shows the results of the priming score calculated by subtracting the mean RTs to trained sequences from untrained sequences for positions 3-6; these values were averaged across positions 3 and 4 and across positions 5 and 6 (Shanks et al., 2006). Repeated measures $t$ tests performed against chance $(0)$ revealed significant priming in the cTBS $M 1_{\text {HAND }}$ condition for positions 3-4 (24 $\mathrm{ms})$ and $5-6(27 \mathrm{~ms})\left(t_{(7)}=5.62\right.$ and 3.50 , respectively, $p$ values $<0.01$ ), whereas priming was absent in both collapsed locations for cTBS AG and control conditions ( $p$ values $>0.05$ ).

In summary, both the cued-generation task and recognition test were in agreement in revealing evidence of sequence knowledge that was available to conscious awareness in the cTBS AG 


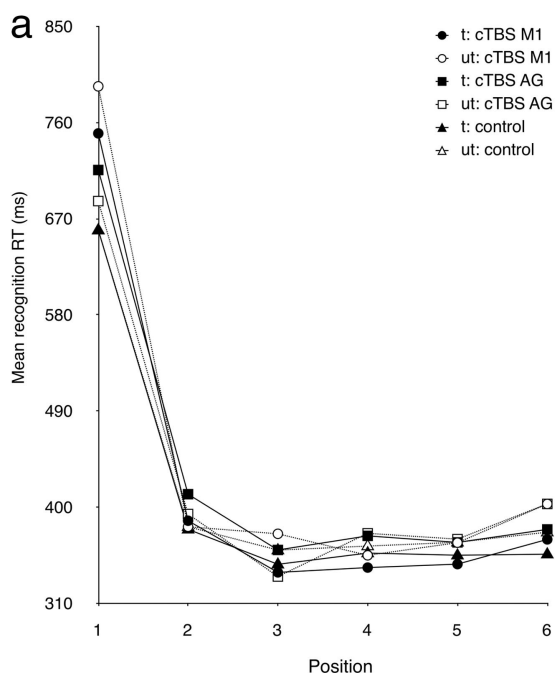

b

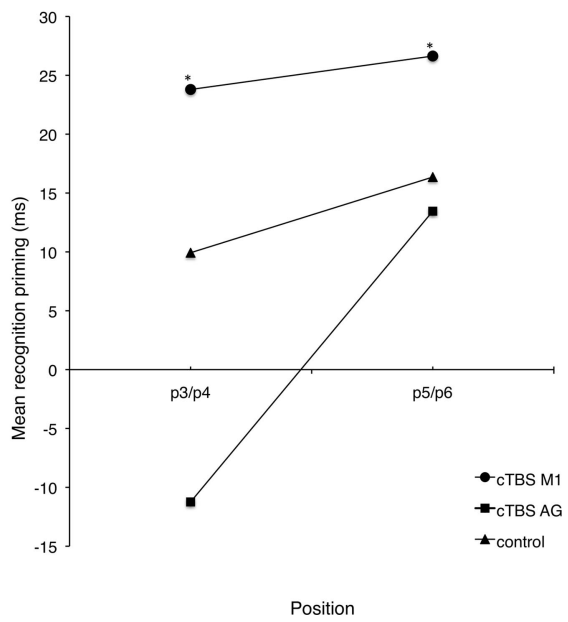

Figure 4. Manual sequence learning (experiment 1). $\boldsymbol{a}$, Mean reaction times to targets associated with trained ( $\mathrm{t}$ ) and untrained (ut) sequences in the manual recognition test. Positions 3-6 were predictable from the preceding targets on trained sequences, whereas positions 1-2 were not predictable. $\boldsymbol{b}$, Mean priming scores (RTs associated with responses to trained positions subtracted from the corresponding RTs to untrained positions) derived from the recognition priming data. Results are combined and presented for positions 3-4 (p3/p4) and 5-6 (p5/p6). Position 3-4 represents the earliest point at which the response context can induce priming. ${ }^{*} p<0.05$.

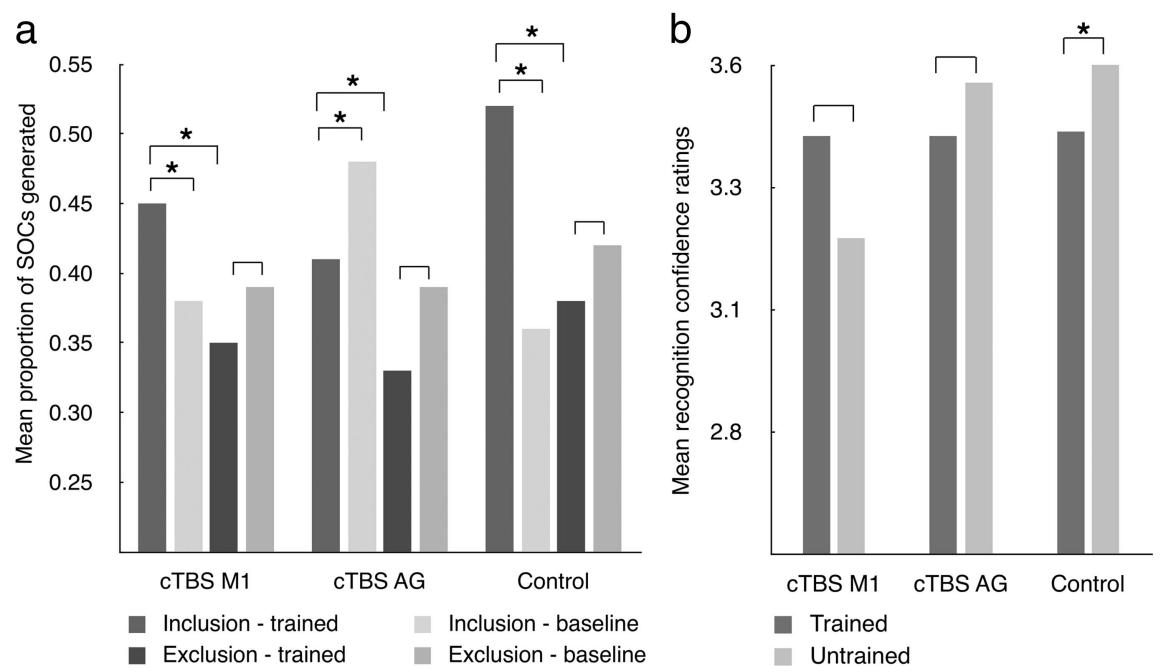

Figure 5. Perceptual sequence learning (experiment 2). $\boldsymbol{a}$, Mean proportions of trained and baseline SOC triplets generated for each site of stimulation condition, under inclusion and exclusion perceptual cued-generation test instructions. $\boldsymbol{b}$, Mean recognition confidence ratings assigned to the 24 six-item short sequences, under each site of stimulation condition. Trained sequences were allocated a rating between 1 and 3 , whereas untrained sequences were allocated a rating between 4 and $6 .{ }^{*} p<0.05$.

(and control) condition but implicit knowledge in the cTBS M1 condition. These data also indicate that, even though concurrent tasks can limit the availability of conscious knowledge (Shanks and Channon, 2002), the vigilance task did not preclude the acquisition of above-baseline knowledge and conscious awareness in the cTBS AG and control condition of experiment 1.

\section{Experiment 2}

Participants in all three between-subject conditions achieved $>95 \%$ accuracy on the vigilance task, which indicates that participants were able to attend consistently to the sequence of target stimuli and could reliably discriminate LDTs from standard targets using covert reorienting of visuospatial attention. Mean end times in the cTBS AG, cTBS $M 1_{\text {HAND }}$, and control conditions were $39 \min 23 \mathrm{~s}, 38 \mathrm{~min} 53 \mathrm{~s}$, and $38 \mathrm{~min} 7 \mathrm{~s}$, respectively.
Effects of cTBS over M1 and AG on posttraining direct tests of learning and awareness

Subjective measures of sequence knowledge. Learning on the perceptual SRT task did not lead to accurate sequence knowledge above the subjective threshold: participants exhibited little or no metaknowledge about the target sequence on the awareness questionnaire in the cTBS $M 1_{\text {HAND }}($ mean $\pm \mathrm{SE}, 1.5 \pm 0.03)$, cTBS $\mathrm{AG}$ (mean $\pm \mathrm{SE}, 1.3 \pm 0.03$ ), and control (mean \pm SE, $1.1 \pm 0.03$ ) conditions, and the level of awareness was equivalent across conditions (Kruskal-Wallis ANOVA, $\chi_{(2)}^{2}=$ $0.51, p=0.78)$. On the cued-generation task, participants were again more confident in their ability to generate a novel sequence on the exclusion test (mean \pm SE, $51.1 \pm 4.45$ ) than generate the trained sequence on the inclusion test (mean $\pm \mathrm{SE}, 27.4 \pm 4.28$ ) $\left(F_{(1,21)}=15.47, p=0.001\right)$, and this was consistent across between-subject conditions $\left(F_{(2,21)}=1.17, p=0.33\right)$.

Cued-generation task. Mean start times in the cTBS AG, cTBS M1 $1_{\text {HAND }}$, and control conditions were $47 \mathrm{~min} 33 \mathrm{~s}, 48 \mathrm{~min}$ $17 \mathrm{~s}$, and $46 \mathrm{~min} 39 \mathrm{~s}$, respectively. Figure $5 a$ shows the mean proportions of trained SOCs generated under each site condition for the inclusion and exclusion tests. A 3 ( site: $\mathrm{cTBS} M 1_{\mathrm{HAND}}, \mathrm{cTBS} \mathrm{AG}$, control) $\times$ 2 (test: inclusion, exclusion) $\times 2$ (sequence: trained, baseline) mixed-factorial ANOVA performed on the mean proportions of second-order triplets generated revealed a significant main effect of test $\left(F_{(1,21)}=46.24, p<0.001\right)$ and significant interactions between site and sequence $\left(F_{(2,21)}=6.84, p<0.01\right)$, between test and sequence $\left(F_{(1,21)}=17.01, p<0.001\right)$, and between site, test, and sequence $\left(F_{(2,21)}=\right.$ 7.07, $p<0.01)$. In the perceptual cTBS AG condition, although there was a significant difference in the proportion of trained triplets generated on the inclusion relative to exclusion test $\left(F_{(1,21)}=7.62\right.$, $p<0.01$ ), this difference was undermined by the failure to exceed baseline performance on the inclusion [0.41 (trained) vs 0.48 (baseline) $]$ and exclusion $[0.33$ (trained) vs 0.38 (baseline) $]$ tests $\left(F_{(1,21)}=8.98\right.$, $p<0.01$ and $F_{(1,21)}=2.38, p=0.14$, respectively). In the cTBS $M 1_{\text {HAND }}$ condition, conscious control when responding with trained sequences (i.e., I $>$ E) $\left(F_{(1,21)}=13.09, p<0.01\right)$ was accompanied by above-baseline performance on the inclusion test but not on the exclusion test $\left(F_{(1,21)}=8.98, p<0.01\right.$ and $F_{(1,21)}=1.42, p=0.25$, respectively). Participants in the control condition were also able to exert conscious control when responding with the trained sequence (i.e., I trained $>$ E trained) $\left(F_{(1,21)}=28.45\right.$, $p<0.0001)$, and performance was above and equivalent to baseline on the inclusion and exclusion tests $\left(F_{(1,21)}=35.93, p<0.0001\right.$ and $F_{(1,21)}=1.59, p=0.22$, respectively). Collectively, these results indicate that sequence knowledge in the perceptual cTBS AG 
condition was absent [I $(\mathrm{I}$ trained $<\mathrm{B})>\mathrm{E}(\mathrm{E}$ trained $=\mathrm{B})$ ], whereas knowledge was available to support conscious generation of the trained sequence in the cTBS $\mathrm{M} 1_{\mathrm{HAND}}$ and control conditions (I trained $>$ E trained; I trained $>$ B; E trained $=\mathrm{B}$ ). All planned comparisons were significant for Bonferroni-Holm corrected $p$ values.

Recognition confidence ratings. Mean start times in the cTBS AG, cTBS M1 $1_{\text {HAND }}$, and control conditions were 61 min $54 \mathrm{~s}, 60$ min $66 \mathrm{~s}$, and $59 \mathrm{~min} 38 \mathrm{~s}$, respectively. Mean recognition confidence ratings to trained and untrained sequences recognition for each site of stimulation condition are shown in Figure $5 b$. A 3 (site: $\mathrm{M} 1_{\text {HAND }}, \mathrm{AG}$, control) $\times 2$ (sequence: trained, untrained) mixed-factorial ANOVA revealed a significant interaction between site and sequence $\left(F_{(2,21)}=3.08, p<0.05\right)$. Importantly, planned comparisons revealed that participants in the control condition could discriminate between trained and untrained sequences $\left(F_{(1,21)}=6.07, p<0.05\right)$, whereas participants in the AG and $\mathrm{M} 1_{\text {HAND }}$ conditions were at chance $\left(F_{(1,21)}=0.44, p=0.51\right.$ and $F_{(1,21)}=2.05, p=0.17$, respectively). Hence, the results from the recognition test revealed that perceptual learning led to explicit knowledge in the control condition. Although results from the cued-generation task and recognition test were not in agreement in the cTBS $M 1_{\text {HAND }}$ condition, it is important to note that functional dissociations between subjective and objective measure of learning and awareness have been reported in other studies [e.g., forced-choice questionnaire vs free-generation and recognition (Destrebecqz and Cleeremans, 2003)] and are variously argued to reveal gradations in the awareness with which sequence knowledge is held (Norman et al., 2006) or differences in the nature of knowledge applied when responding on different measures (Dienes et al., 1995). None of these interpretations, however, is at variance with the notion that explicit knowledge on the cued-generation task in the cTBS $\mathrm{M} 1_{\mathrm{HAND}}$ condition provides evidence of conscious awareness.

\section{Discussion}

We investigated the cortical substrate underlying manual and perceptual probabilistic sequence learning by administering cTBS over M1 and the right AG. Four novel results were obtained: (1) cTBS over M1 and AG led to a functional-temporal dissociation in manual sequence learning: learning was disrupted across $75 \%$ trials in the cTBS M1 condition, whereas in the cTBS AG condition, disruption was confined to a midpoint during training; (2) cTBS over M1 modulated awareness associated with manual sequence learning: posttraining direct tests revealed that sequence knowledge was implicit in the cTBS M1 condition, whereas sequence knowledge was explicit in the cTBS AG and control conditions; (3) covert reorienting of visuospatial attention to visual stimuli supported probabilistic sequence learning; and (4) perceptual sequence learning was abolished by cTBS administered over the right $\mathrm{AG}$.

\section{Functional-temporal dissociation between M1 and AG during manual sequence learning}

cTBS over M1 led to interference with learning for at least nine training blocks ( 23 min $52 \mathrm{~s}$ ), whereas the effect of cTBS over the AG led to loss of sensitivity to improbable trials that peaked at a midpoint during training. Interestingly, the chronometry of the effect in the M1 condition corresponds with the timing of early activation associated with consolidation mechanisms implicated in manual skill and sequence learning (Muellbacher et al., 2002; Seidler et al., 2005), and is broadly in line with the duration of the suppressive effect of cTBS over M1 on motor-evoked potentials
(20 s cTBS/20 min effect) (Huang et al., 2005; Reis al., 2008). TBS-induced functional effects after stimulation over the parietal cortex have thus far been associated with tonic, rather than phasic, effects lasting up to $30 \mathrm{~min}$ (Nyffeler et al., 2008).

Importantly, given that the SRT task error rate was comparable across conditions, the effects were specific to learning and not the result of impairment with response execution or a speedaccuracy tradeoff and are likely to have involved disruption of effector-independent and effector-dependent learning (Bapi et al., 2000). Results in the cTBS M1 condition are thus consistent with evidence showing that procedural aspects of learning on the manual SRT task exhibit relative insensitivity to posttraining TMS over M1 (Goedert and Willingham, 2002; Press et al., 2005). Disentangling learning from performance is important because changes in motor performance alone can lead to changes in the activity of neural areas implicated in learning. Compensatory mechanisms involving the recruitment of other parts of the motor system could, however, have masked interference with response execution (Lee et al., 2003). Indeed, coactivation and/or inhibition of topographically and functionally related regions, such as the dorsal premotor cortex (PMd), supplementary motor area (SMA), cingulate motor cortex, thalamus, putamen, and contralateral M1 (Chouinard et al., 2003; Bestmann et al., 2004), are possible because TMS induces effects proximal and distal to the site of stimulation (Hallett, 2000).

Although subregions such as PMd do not have a readily identifiable casual role in manual sequence learning beyond a contribution to the selection of movements according to learned arbitrary rules (Thoenissen et al., 2002; Perez et al., 2007), sequence learning is routinely associated with coherent preferential activation of cortico-cerebellar and cortico-striatal networks in early and late phases (and delayed recall) of manual learning, respectively (Doyon and Ungerleider, 2002; Doyon et al., 2003). Subregions that support early motor skill learning exhibit a temporal gradient in activation: a decrease in cerebellar activation with learning is accompanied by an increase in activation of the basal ganglia, M1, and the SMA during later stages (Hazeltine et al., 1997; Grafton et al., 1998b). Interestingly, the production, rather than learning, of sequences is identified with the SMA (Perez et al., 2007; Schwarb and Schumacher, 2009), whereas M1 has been implicated in procedural consolidation (Muellbacher et al., 2002), whereby a learned skill becomes less susceptible to interference (Robertson et al., 2004). Given this role, it was important to assess the implications of cTBS-induced interference on the nature of newly acquired sequence knowledge using the declarative tests.

\section{Motor-response-dependent contribution of M1 to the state of awareness associated with sequence knowledge}

Learning in the cTBS M1 condition was characterized by a lack of awareness of the learning process and its contents, thereby meeting the conservative criterion for implicit knowledge (Cleeremans, 1993). Participants failed to detect the sequence on the questionnaire but generated comparable, above-baseline, trained sequence elements on the cued-generation tests and two elements primed responses on the recognition test without recognition. In contrast, performance in the control and cTBS AG conditions was consistent with explicit learning: conscious generation and recognition of the trained sequence accompanied awareness on the questionnaire. M1 thus has a causal role in developing experience-dependent functional attributes relevant to conscious sequence knowledge, and this role appears to be motor response dependent; in experiment 2, there was a null 
effect of cTBS over M1 on the state of awareness associated with perceptual sequence knowledge, albeit after a longer duration of cTBS over M1 (30 vs 20 s).

Our results are in agreement with TMS studies that have documented an effector-specific role for the M1 in manual sequence learning (Pascual-Leone et al., 1994, 1996). Previous studies indicate that M1 is involved with the representation of parameters beyond movement dynamics and kinematics, such as serial order or movement context (Grafton et al., 1998b; Carpenter et al., 1999; Matsuzaka et al., 2007). Multiple additional sources of information contribute to manual sequence learning (Verwey and Wright, 2004), such as coding for the movement or goal of the movement (Grafton et al., 1998a). Explicit motor response information is hypothesized to involve the integration and organization of action-effect codes into an ordered plan of actions (Hazeltine, 2002; Tubau et al., 2007). Importantly, learning-related changes in the motor cortices involve modulation from prefrontal cortex and posterior parietal cortex (PPC) in forming abstract goal and/or response-based representations (Shadmehr and Krakauer, 2008). Such abstract representations imply a level of global access in which information can be deployed flexibly in response to varying contextual demands, which is, arguably, a fundamental feature of consciousness (Baars, 2002). Hence, the modulation in awareness was arguably attributable to a loss of, or impairment with, effector and/or response-specific information that had an impact on the formation of such representations and on perceptualmotor integration processes involved with developing knowledge that can be deployed flexibly.

\section{The angular gyrus had a critical role in perceptual but not manual sequence learning}

In the perceptual cTBS M1 and control conditions, rapid-occurring within $100 \mathrm{~ms}$ of a predictive cue (Lambert, 2003) - and sustained covert reorienting of visuospatial attention rather than action supported probabilistic sequence learning. Perceptual knowledge was expressed through the conscious generation and recognition of the trained sequence. These data serve as a counterpoint to accounts in which motor responses are a condition for sequence learning (Deroost and Soetens, 2006) but are consistent with the view that perceptual learning involves conscious access (Knee et al., 2007) and elaborate on previous work by demonstrating that perceptual learning can proceed without an intentional orientation during training. Importantly, perceptual learning was abolished by cTBS over the AG: there was no evidence of sequence knowledge on the cued-generation and recognition tests. In contrast, performance on the manual cued-generation and recognition test was consistent with explicit knowledge. Involvement of the right AG in sequence learning was thus sensitive to the mode of response.

Failure to detect learning in the perceptual cTBS AG condition did not emerge because perception of visual stimuli was impaired; performance on the vigilance task was at ceiling in all conditions. This is an important consideration because TMS administered over the right posterior parietal cortex can interfere with spatial orienting and attentional processes and induce neglect-like behavior (Hilgetag et al., 2001; Rosenthal et al., 2006; Rounis et al., 2007). Although the right AG has a particular role in the reorienting of spatial attention (Chambers et al., 2004), visuospatial orienting of attention depends on a network of frontal and parietal areas in the right hemisphere that extends to bilateral hemispaces (Corbetta and Shulman, 2002; Husain and Nachev, 2007). Because focal lesions within this network lead to discrete functional deficits, it will be important to examine the effect that disruption has on perceptual learning. For example, the lateral prefrontal cortex is instrumental in orienting attention, particularly in relation to the selection of task-relevant information (Brass and von Cramon, 2004), as part of an anterior striatum basal ganglia loop involved in visuospatial representation (Hikosaka et al., 2002), whereas neurons within the lateral intraparietal area in nonhuman primates have been shown to integrate probabilistic evidence regarding spatial positions (Yang and Shadlen, 2007).

Although the foci, and lateralization, of activation within the parietal cortex during manual sequence learning vary across functional MRI studies (Hazeltine et al., 1997; Grafton et al., 1998b; Willingham et al., 2002; Schendan et al., 2003; BischoffGrethe et al., 2004; Olson et al., 2006), activation within the parietal cortex is reported consistently. Manual learning might also be predicted to be susceptible to parietal stimulation on the grounds that changes in ipsilateral M1 are associated with TMS over the PPC (Koch et al., 2007). Nonetheless, our enhanced direct and indirect test protocol revealed comparable manual learning between the cTBS AG and control conditions. Hence, the contribution of the right $A G$ to sequence learning per se did not operate independently of response mode. Previous investigations have similarly reported a null effect of TMS over parietal cortex on manual sequence learning (Robertson et al., 2001), and intact learning on the manual SRT task has been reported in patients with lesions involving the right parietal lobe (Berryhill et al., 2008).

Activation within the right IPL on the manual SRT task thus appears to be a consequence of more generalized processes involving the covert allocation and redirection of attention rather than sequence learning per se. Interestingly, deficits in spatiotemporal organization on tasks involving the predictive remapping of sequential movements occur in patients with ideomotor limb apraxia resulting from lesions within the left parietal cortex (Harrington and Haaland, 1992; Rushworth et al., 1997). Although activation within left inferior parietal cortex during the manual SRT task is associated with changes in performance rather than sequence learning per se (Willingham et al., 2002), disruption of the left parietal lobe contribution to motor attention might influence manual sequence learning (Rushworth et al., 2001). In particular, activation within the left supramarginal gyrus is associated with the covert preparation of movements, which, in the manual SRT task, is likely to be related to constant redirections of motor attention (Rushworth et al., 2003).

\section{Commonalities and differences between motor and perceptual sequence learning}

Comparable training parameters were implemented across the manual and perceptual SRT tasks, but differences along dimensions such as stimulus-response ( $\mathrm{S}-\mathrm{R}$ ) compatibility are likely to influence learning. Lower relative $\mathrm{S}-\mathrm{R}$ compatibility in the manualattributable to greater response selection demands (Sternberg, 1969) - relative to the perceptual SRT task is likely to facilitate sequence learning (Koch and Hoffman, 2000). Sensitivity to changes in S-R compatibility has been observed within the PPC, including the IPL, left SMA, premotor cortex, and anterior cingulate cortex (Nee et al., 2007). It will be important to explore whether there are any differences between manual and perceptual learning in the effect of cTBS on the superior parietal cortex because this area, along with the PMd and dorsal prefrontal cortex, mediates spatial response selection (Dassonville et al., 2001; Jiang and Kanwisher, 2003; Schumacher et al., 2007) and the formation of spatial cue-to-response associations (Wise et al., 
1997) and is consistently activated on the manual SRT task (Grafton et al., 2002; Olson et al., 2006).

\section{Conclusion}

The present results point to a functional-temporal dissociation between M1 and AG during manual sequence learning that had direct implications for the conscious accessibility of sequence knowledge. Training of covert attention to spatial locations led to probabilistic sequence learning, which was dependent on intact function within the IPL. Differences between the manual and perceptual SRT tasks along dimensions such as response-to-stimulus learning and integrated spatial/stimulus-response-based information were sufficient to modulate the contributions of $\mathrm{M} 1$ and $\mathrm{AG}$ to sequence learning. We conclude that the AG had a critical role in perceptual sequence learning and disruption of the AG did not affect the conscious expression of sequence knowledge in manual sequence learning, whereas M1 had a causal role in developing experience-dependent functional attributes relevant to conscious sequence knowledge on manual but not perceptual sequence learning.

\section{References}

Albouy G, Sterpenich V, Balteau E, Vandewalle G, Desseilles M, Dang-Vu T, Darsaud A, Ruby P, Luppi PH, Degueldre C, Peigneux P, Luxen A, Maquet P (2008) Both the hippocampus and striatum are involved in consolidation of motor sequence memory. Neuron 58:261-272.

Baars BJ (2002) The conscious access hypothesis: origins and recent evidence. Trends Cogn Sci 6:47-52.

Bapi RS, Doya K, Harner AM (2000) Evidence for effector independent and dependent representations and their differential time course of acquisition during motor sequence learning. Exp Brain Res 132:149-162.

Berryhill ME, Mazuz YS, Olson IR (2008) Serial reaction time performance following right parietal lobe damage. J Neuropsychol 2:509-514.

Bestmann S, Baudewig J, Siebner HR, Rothwell JC, Frahm J (2004) Functional MRI of the immediate impact of transcranial magnetic stimulation on cortical and subcortical motor circuits. Eur J Neurosci 19:1950-1962.

Bischoff-Grethe A, Goedert KM, Willingham DT, Grafton ST (2004) Neural substrates of response-based sequence learning using fMRI. J Cogn Neurosci 16:127-138.

Brass M, von Cramon DY (2004) Selection for cognitive control: a functional magnetic imaging study on the selection of task-relevant information. J Neurosci 24:8847-8852.

Carpenter AF, Georgopoulos AP, Pellizzer G (1999) Motor cortical encoding of serial order in a context-recall task. Science 283:1752-1757.

Chambers CD, Payne JM, Stokes MG, Mattingley JB (2004) Fast and slow parietal pathways mediate spatial attention. Nat Neurosci 7:217-218.

Cheeran B, Talelli P, Mori F, Koch G, Suppa A, Edwards M, Houlden H, Bhatia K, Greenwood R, Rothwell JC (2008) A common polymorphism in the brain-derived neurotrophic factor gene (BDNF) modulates human cortical plasticity and the response to rTMS. J Physiol 586:5717-5725.

Chouinard PA, Van Der Werf YD, Leonard G, Paus T (2003) Modulating neural networks with transcranial magnetic stimulation applied over the dorsal premotor and primary motor cortices. J Neurophysiol 90:1071-1083.

Cleeremans A (1993) Mechanisms of implicit learning: a connectionist model of sequence processing Cambridge, MA: Massachusetts Institute of Technology.

Clegg BA, DiGirolamo GJ, Keele SW (1998) Sequence learning. Trends Cogn Sci 2:275-281.

Corbetta M, Shulman GL (2002) Control of goal-oriented and stimulusdriven attention in the brain. Nat Rev Neurosci 3:201-215.

Curran T (1997) Effects of aging on implicit sequence learning: accounting for sequence structure and explicit knowledge. Psychol Res 60:24-41.

Dassonville P, Lewis SM, Zhu XH, Ugurbil K, Kim SG, Ashe J (2001) The effect of stimulus-response compatibility on cortical motor activation. Neuroimage 13:1-14.

Deroost N, Soetens E (2006) Perceptual or motor learning in SRT tasks with complex sequence structures. Psychol Res 70:88-102.

Destrebecqz A, Cleeremans A (2001) Can sequence learning be implicit? New evidence with the process dissociation procedure. Psychon Bull Rev 8:343-350.
Destrebecqz A, Cleeremans A (2003) Temporal effects in sequence learning. In: Attention and implicit learning (Jimenez L, ed), pp 181-214. Amsterdam: Benjamins.

Dienes Z, Altmann GTM, Kwan L, Goode A (1995) Unconscious knowledge of artificial grammars is applied strategically. J Exp Psychol Learn Mem Cogn 21:1322-1338.

Doyon J, Ungerleider L (2002) Functional anatomy of motor skill learning. In: Neuropsychology of memory (Squire LR, Schacter DL, eds), pp 225238. New York: Guildford.

Doyon J, Penhune V, Ungerleider LG (2003) Distinct contributions of the cortico-striatal and cortico-cerebellar systems to motor skill learning. Neuropsychologia 41:252-262.

Goedert KM, Willingham DB (2002) Patterns of interference in sequence learning and prism adaptation inconsistent with the consolidation hypothesis. Learn Mem 9:279-292.

Grafton ST, Fagg AH, Arbib MA (1998a) Dorsal premotor cortex and condition movement selection: a PET functional mapping study. J Neurophysiol 79:1092-1097.

Grafton ST, Hazeltine E, Ivry RB (1998b) Abstract and effector-specific representations of motor sequences identified with PET. J Neurosci 18:9420-9428.

Grafton ST, Hazeltine E, Ivry RB (2002) Motor sequence learning with the nondominant left hand: a PET functional imaging study. Exp Brain Res $146: 369-378$

Hallett M (2000) Transcranial magnetic stimulation and the human brain. Nature 406:147-150.

Harrington DL, Haaland KY (1992) Motor sequencing with left hemisphere damage: are some cognitive deficits specific to limb apraxia? Brain 115: $857-874$

Hazeltine E (2002) The representational nature of sequence learning: Evidence for goal-based codes. In: Common mechanisms in perception and action: attention and performance (Prinz W, Hommel B, eds), pp 673689. Oxford: Oxford UP.

Hazeltine E, Grafton ST, Ivry R (1997) Attention and stimulus characteristics determine the locus of motor sequence encoding: a PET study. Brain 120:123-140.

Hikosaka O, Nakamura K, Sakai K, Nakahara H (2002) Central mechanisms of motor skill learning. Curr Opin Neurobiol 12:217-222.

Hilgetag CC, Théoret H, Pascual-Leone A (2001) Enhanced visual spatial attention ipsilateral to rTMS-induced "virtual lesions" of human parietal cortex. Nat Neurosci 4:953-957.

Huang YZ, Edwards MJ, Rounis E, Bhatia KP, Rothwell JC (2005) Theta burst stimulation of the human motor cortex. Neuron 45:201-206.

Huang YZ, Rothwell JC, Edwards MJ, Chen RS (2008) Effect of physiological activity on an NMDA-dependent form of cortical plasticity in human. Cereb Cortex 18:563-570.

Husain M, Nachev P (2007) Space and the parietal cortex. Trends Cogn Sci 11:30-36.

Jasper H (1958) Report of the committee on methods of clinical examination in electroencephalography. Electroencephalogr Clin Neurophysiol 10:370-375.

Jiang Y, Kanwisher N (2003) Common neural substrate for response selection across modalities and mapping paradigms. J Cogn Neurosci 15:1080-1094.

Juola JF, Koshino H, Warner CB (1995) Tradeoffs between attentional effects of spatial cues and abrupt onsets. Percept Psychophys 57:333-342.

Knee R, Thomason S, Ashe J, Willingham DT (2007) The representation of explicit motor sequence knowledge. Mem Cognit 35:326-333.

Koch G, Fernandez Del Olmo M, Cheeran B, Ruge D, Schippling S, Caltagirone C, Rothwell JC (2007) Focal stimulation of the posterior parietal cortex increases the excitability of the ipsilateral motor cortex. J Neurosci 27:6815-6822.

Koch I, Hoffman J (2000) The role of stimulus-based and response-based spatial information in sequence learning. J Exp Psychol Learn Mem Cogn 26:863-882.

Lambert A (2003) Visual orienting, learning and conscious awareness. In: Attention and implicit learning, pp 253-275. Amsterdam: Benjamins.

Lee L, Siebner HR, Rowe JB, Rizzo V, Rothwell JC, Frackowiak RS, Friston KJ (2003) Acute remapping within the motor system induced by lowfrequency repetitive transcranial magnetic stimulation. J Neurosci 23:5308-5318.

Majerus S, Poncelet M, Van der Linden M, Albouy G, Salmon E, Sterpenich V, 
Vandewalle G, Collette F, Maquet P (2006) The left intraparietal sulcus and verbal short-term memory: focus of attention or serial order. Neuroimage 32:880-891.

Matsuzaka Y, Picard N, Strick PL (2007) Skill representation in the primary motor cortex after long-term practice. J Neurophysiol 97:1819-1832.

Muellbacher W, Ziemann U, Wissel J, Dang N, Kofler M, Facchini S, Boroojerdi B, Poewe W, Hallett M (2002) Early consolidation in human primary motor cortex. Nature 415:640-644.

Nee DE, Wager TD, Jonides J (2007) Interference resolution: insights from a meta-analysis of neuroimaging tasks. Cogn Affect Behav Neurosci 7:1-17.

Nissen MJ, Bullemer P (1987) Attentional requirements of learning: evidence from performance measures. Cogn Psychol 19:1-32.

Norman E, Price MC, Duff SC (2006) Fringe consciousness in sequence learning: the evidence of individual differences. Conscious Cogn 15:723-760.

Nyffeler T, Cazzoli D, Wurtz P, Lüthi M, von Wartburg R, Chaves S, Déruaz A, Hess CW, Müri RM (2008) Neglect-like visual exploration behaviour after theta burst transcranial magnetic stimulation of the right posterior parietal cortex. Eur J Neurosci 27:1809-1813.

Oldfield RC (1971) The assessment and analysis of handedness: the Edinburgh inventory. Neuropsychologia 9:97-113.

Olson IR, Rao H, Moore KS, Wang J, Detre JA, Aguirre GK (2006) Using perfusion fMRI to measure continuous changes in neural activity with learning. Brain Cogn 60:262-271.

Pascual-Leone A, Grafman J, Hallett M (1994) Modulation of cortical motor output maps during development of implicit and explicit knowledge. Science 263:1287-1289.

Pascual-Leone A, Wassermann EM, Grafman J, Hallett M (1996) The role of the prefrontal cortex in implicit procedural learning. Exp Brain Res 107:479-485.

Perez MA, Tanaka S, Wise SP, Sadato N, Tanabe HC, Willingham DT, Cohen LG (2007) Neurla substrates of intermanual transfer of a newly acquired motor skill. Curr Biol 17:1896-1902.

Press DZ, Casement MD, Pascual-Leone A, Robertson EM (2005) The time course of off-line motor sequence learning. Cogn Brain Res 25:375-378.

Reed J, Johnson P (1994) Assessing implicit learning with indirect tests: determining what is learned about sequence structure. J Exp Psychol Learn Mem Cogn 20:585-594.

Reis J, Robertson E, Krakauer JW, Rothwell J, Marshall L, Gerloff C, Wassermann E, Pascual-Leone A, Hummel F, Celnik PA, Classen J, Floel A, Ziemann U, Paulus W, Siebner HR, Born J, Cohen LG (2008) Consensus: can tDCS and TMS enhance motor learning and memory formation? Brain Stimulat 1:363-369.

Robertson EM, Tormos JM, Maeda F, Pascual-Leone A (2001) The role of the dorsolateral prefrontal cortex during sequence learning is specific for spatial information. Cereb Cortex 11:628-635.

Robertson EM, Théoret H, Pascual-Leone A (2003) Studies in cognition: the problems solved and created by transcranial magnetic stimulation. J Cogn Neurosci 15:948-960.

Robertson EM, Pascual-Leone A, Miall RC (2004) Current concepts in procedural consolidation. Nat Rev Neurosci 5:576-582.

Rosenthal CR, Walsh V, Mannan SK, Anderson EJ, Hawken MB, Kennard C (2006) Temporal dynamics of parietal cortex involvement in visual search. Neuropsychologia 44:731-743.

Rounis E, Yarrow K, Rothwell JC (2007) Effects of rTMS conditioning over the fronto-parietal network on motor versus visual attention. J Cogn Neurosci 19:513-524.

Rushworth MFS, Nixon PD, Renowden S, Wade DT, Passingham RE (1997) The left parietal cortex and motor attention. Neuropsychologia 35:12611273.

Rushworth MFS, Krams M, Passingham RE (2001) The attentional role of the left parietal cortex: the distinct lateralization and localization of motor attention in the human brain. J Cogn Neurosci 13:698-710.

Rushworth MFS, Johansen-Berg H, Gobel SM, Devlin JT (2003) The left parietal and premotor cortices: motor attention and selection. Neuroimage 20 [Suppl 1]:S89-S100.

Sack AT (2009) Parietal cortex and spatial cognition. Behav Brain Res 202:153-161.

Sack AT, Cohen Kadosh R, Schuhmann T, Moerel M, Walsh V, Goebel R (2009) Optimizing functional accuracy of TMS in cognitive studies: a comparison of methods. J Cogn Neurosci 21:207-221.

Schendan HE, Searl MM, Melrose RJ, Stern CE (2003) An fMRI study of the role of the medial temporal lobe in implicit and explicit sequence learning. Neuron 37:1013-1025.

Schumacher EH, Cole MW, D'Esposito M (2007) Selection and maintenance of stimulus-response rules during preparation and performance of a spatial choice-reaction task. Brain Res 1136:77-87.

Schwarb H, Schumacher EH (2009) Neural evidence of a role for spatial response selection in the learning of spatial sequences. Brain Res 1247:114-125.

Seidler RD, Purushotham A, Kim SG, Ugurbil K, Willingham D, Ashe J (2005) Neural correlates of encoding and expression in implicit sequence learning. Exp Brain Res 165:114-124.

Shadmehr R, Krakauer JW (2008) A computational neuroanatomy for motor control. Exp Brain Res 185:359-381.

Shanks DR (2005) Implicit learning. In: Handbook of cognition (Lamberts K, Goldstone R, eds), pp 202-220. London: Sage.

Shanks DR, Channon S (2002) Effects of a secondary task on "implicit" sequence learning: learning or performance? Psychol Res 66:99-109.

Shanks DR, Johnstone T (1999) Evaluating the relationship between explicit and implicit knowledge in a sequential reaction time task. J Exp Psychol Learn Mem Cogn 25:1435-1451.

Shanks DR, Perruchet P (2002) Dissociation between priming and recognition in the expression of sequential knowledge. Psychon Bull Rev 9:362-367.

Shanks DR, Channon S, Wilkinson L, Curran HV (2006) Disruption of sequential priming in organic and pharmacological amnesia: a role for the medial temporal lobes in implicit contextual learning. Neuropsychopharmacology 31:1768-1776.

Song S, Howard JH Jr, Howard DV (2008) Perceptual sequence learning in a serial reaction time task. Exp Brain Res 189:145-158.

Sternberg S (1969) The discovery of processing stages: extensions of Donders' method. Acta Psychologica 30:276-315.

Thoenissen D, Zilles K, Toni I (2002) Differential involvement of parietal and precentral regions in movement preparation and motor intention. J Neurosci 22:9024-9034.

Tubau E, Hommel B, López-Moliner J (2007) Modes of executive control in sequence learning: from stimulus-based to plan-based control. J Exp Psychol Gen 136:43-63.

Verwey WB, Wright DL (2004) Effector-independent and effectordependent learning in the discrete sequence production task. Psychol Res 68:64-70.

Wilkinson L, Jahanshahi M (2007) The striatum and probabilistic implicit sequence learning. Brain Res 1137:117-130.

Willingham DB (1998) A neuropsychological theory of motor skill learning. Psychol Rev 105:558-584.

Willingham DB, Salidis J, Gabrieli JDE (2002) Direct comparison of neural systems mediating conscious and unconscious skill learning. J Neurophysiol 88:1451-1460.

Wise SP, Boussaoud D, Johnson PB, Caminiti R (1997) Premotor and parietal cortex: corticocortical connectivity and combinatorial computations. Annu Rev Neurosci 20:25-42.

Yang T, Shadlen MN (2007) Probabilistic reasoning by neurons. Nature 447:1075-1080.

Ziemann U, Ilić TV, Iliać TV, Pauli C, Meintzschel F, Ruge D (2004) Learning modifies subsequent induction of long-term potentiation-like and long-term depression-like plasticity in human motor cortex. J Neurosci $24: 1666-1672$. 